\title{
Development of Novel RF and Millimeter Wave Structures by Laser Direct-write
}

\author{
Scott A. MATHEWS ${ }^{1}$, Mark MIROTZNIK ${ }^{1}$ and Alberto PIQUÉ ${ }^{2}$ \\ ${ }^{1}$ The Catholic University of America, Washington, DC 20064, USA \\ E-mail: mathewss@cua.edu \\ ${ }^{2}$ Naval Research Laboratory, Washington, DC 20375, USA
}

\begin{abstract}
In this work we describe the use of laser direct-write for the rapid prototyping of frequency selective Fresnel zone plate lenses. We describe a frequency selective surface which is patterned on two distinct length scales; a smaller scale that exhibits the patterning of a traditional frequency selective surface, and a larger scale that exhibits the structure of a Fresnel zone plate. We demonstrate that laser direct-write is an ideal tool for the rapid prototyping of such spatially varying or aperiodic electromagnetic structures. We present experimental results for the electromagnetic characterization of a prototype frequency selective lens fabricated using non-lithographic, laser processing.

DOI: 10.2961/jlmn.2009.03.0006
\end{abstract}

Keywords: Frequency selective surface, laser direct-write, laser microfabrication, rapid prototyping, Fresnel zone plate, non-lithographic patterning

\section{Introduction}

A frequency selective surface (FSS) is a periodic surface that comprises an array of identical elements (i.e. crosses, loops, grids, etc.) repeated in one or two dimensions. A FSS is designed to transmit or reflect electromagnetic radiation in a specific band or bands. The periodic element in a FSS can be a patterned dielectric or a patterned conductor. A FSS comprising conducting elements can be "free standing" (i.e. slots cut into a metal sheet) or bonded to a dielectric layer which acts both to alter the electromagnetic properties and to provide a semi-rigid support (i.e. copper elements patterned on high frequency circuit board material). Single layers of patterned dielectric or conductor can be stacked or laminated to form multi-layer FSSs.

A FSS is designed to perform at a specific frequency band or bands. The periodic shapes patterned into the dielectric or conducting layer are generally smaller than the wavelength of the radiation in the operating frequency band. The desired wavelength (or frequency) characteristics of a FSS determines the required features size for patterning the various layers. Conversely, the "design rule" or minimum feature size of a given patterning process limits the maximum useful frequency (minimum wavelength) of a FSS. For example, a FSS operating in the infrared portion of the spectrum requires features on the order of microns to tenths of microns in size, while a microwave FSS might require feature sizes from a few tenths of millimeters to a few centimeters. The minimum useful frequency of a FSS is generally determined by the overall size of the FSS. Because FSSs are periodic structures, the transmission and reflection will be highly dependant on the total number of periods "sampled" by the incident wave. The "ideal" properties of a FSS are only achieved with an infinite number of periods. Finite FSSs exhibit less than ideal performance.

Because FSSs have the ability to selectively transmit or reflect electromagnetic radiation, they are well known and widely used in radio, microwave and radar applications [1].
Traditional applications for FSSs include hybrid radomes (enclosures for radar transmit/receive antennas), dichroic reflectors, band-pass and band-stop filters, reduced radar cross section (RCS) structures, and augmented radar cross section structures [2]. More recently, FSS technology has been applied to RFID tags, collision avoidance systems, EMI protection, satellite communications [3], and altering the radio properties of walls and buildings [4].

The term "laser direct-write" refers to a group of nonlithographic laser based patterning techniques. Laser direct-write includes subtractive processes, such as conventional laser micro-machining and laser exposure of photoresist combined with wet etch techniques. Laser directwrite also includes additive processes, such as laser induced forward transfer (LIFT) and laser printing [5]. These techniques have proved to be useful for the fabrication of prototype FSSs due to the fact that they are fast and flexible [6]. Because these processes do not require mask fabrication, designs can be rapidly altered using only software. Because these techniques are laser based, a wide variety of materials can be patterned with high resolution over large areas.

\section{Background}

Determining the electromagnetic properties of a FSS from its geometry and materials parameters of a FSS is often referred to as solving the "forward problem". Although the physics of FSSs are governed by Maxwell's Equations, coupling of individual elements to their nearest neighbors and the complex geometry of some FSSs make the forward problem impossible to solve in closed form for all but the simplest configurations. More complex configurations can be solved numerically. Several algorithms have been developed that are extremely effective in solving the forward problem. These algorithms have been implemented in a variety of computer languages and software packages, including off-the-shelf software "tool boxes" written specifically for FSSs [7]. Two commonly used al- 
gorithms for solving the forward problem are the "Periodic Method of Moments" (PMM) and the "Finite Difference Time Domain" (FDTD) method.

The periodic method of moments was developed by Ben Munk and his collogues at Ohio State University in the mid-1960's. This approach to solving the forward problem has become the "gold standard" in designing FSSs. Having said that, PMM does have limitations in terms of its ability to handle finite arrays of elements. The FDTD method also has its advantages and disadvantages. While the FDTD method can more easily handle the modeling of finite surfaces, it is computationally more intensive. Both of these techniques require detailed knowledge of the electromagnetic properties of the materials used to fabricate the FSS.

While these methods of solving the forward problem have proven quite effective, at some point the designers of FSSs need to fabricate and measure the performance of the surface. This is done not only to validate the model, but to confirm the values of the materials properties entered into the models (e.g. dielectric constant, conductivity, physical dimensions, etc.). For many materials, the values of dielectric constant and conductivity are not well known at the frequencies in question. Additionally, commercially available materials that are not specifically designed for use in microwave or millimeter wave applications can show significant variation from part to part or batch to batch. Materials that require processing as part of the FSS fabrication (i.e. polymer resins, adhesives, or conducing pastes) can show significant variation in high frequency properties due to variations in cure time, cure temperature, and shelf life. This problem is exacerbated by the fact that novel FSS applications may require the use of a wide variety of nontraditional materials. These materials, although desirable for constructing FSSs, are not specifically designed for microwave or millimeter wave applications, and may therefore have significant uncertainty associated with their high frequency materials properties.

In addition to uncertainty in the materials properties, rapid prototyping of FSSs becomes extremely important when designing spatially varying or aperiodic subwavelength structures. Because each of the FSS elements is sub-wavelength in its physical dimensions, the "cell size", or "voxel" that a numeric model uses to simulate the structure must be relatively small in order to accurately reflect the geometry of the element (i.e. fine mesh discretization). If a structure cannot be described by a periodic boundary condition, the complete structure must be entered into the numerical model. This tends to result in extremely large arrays, and therefore an extremely large number of numeric iterations. Given that both the FDTD and PMM algorithms are already computationally intensive, the increase in array size associated with modeling spatially varying structures may require an inordinate amount of computational time (or computational resources) to converge on a solution. The end result is that many non-traditional FSS structures may require more time and effort to be accurately modeled than to be fabricated using rapid prototyping techniques.

One particular pattern that cannot be modeled with a periodic boundary condition is the frequency selective Fresnel zone plate. A Fresnel zone plate is screen comprised of concentric transparent and opaque rings, as shown in figure 1a. The rings are designed in such a way as to block the transmission of radiation from every other Fresnel half-period zone [8]. This allows the radiation passing through the transparent rings to arrive at a particular point in space with the appropriate phase relationship so as to interfere constructively. The result is that a Fresnel zone plate will act as an extremely thin lens, with a wavelength dependant focal distance, as shown schematically in figure 1b. These types of lenses have been widely used in combination with antennas [9].

In light of the previous discussion, a number of rapid prototyping techniques have been applied to FSSs and subwavelength electromagnetic structures [10], including several laser based techniques [11]. These techniques include both additive and subtractive laser processes, capable of patterning both conductors and dielectrics with resolution on the order of a few microns, over areas as large as a square meter. In this work, we demonstrate the use of one such technique to fabricate a Fresnel zone plate combined with a frequency selective surface to form a frequency selective lens. This structure acts as a lens, focusing electromagnetic energy, only in a narrow band of frequencies.

a)
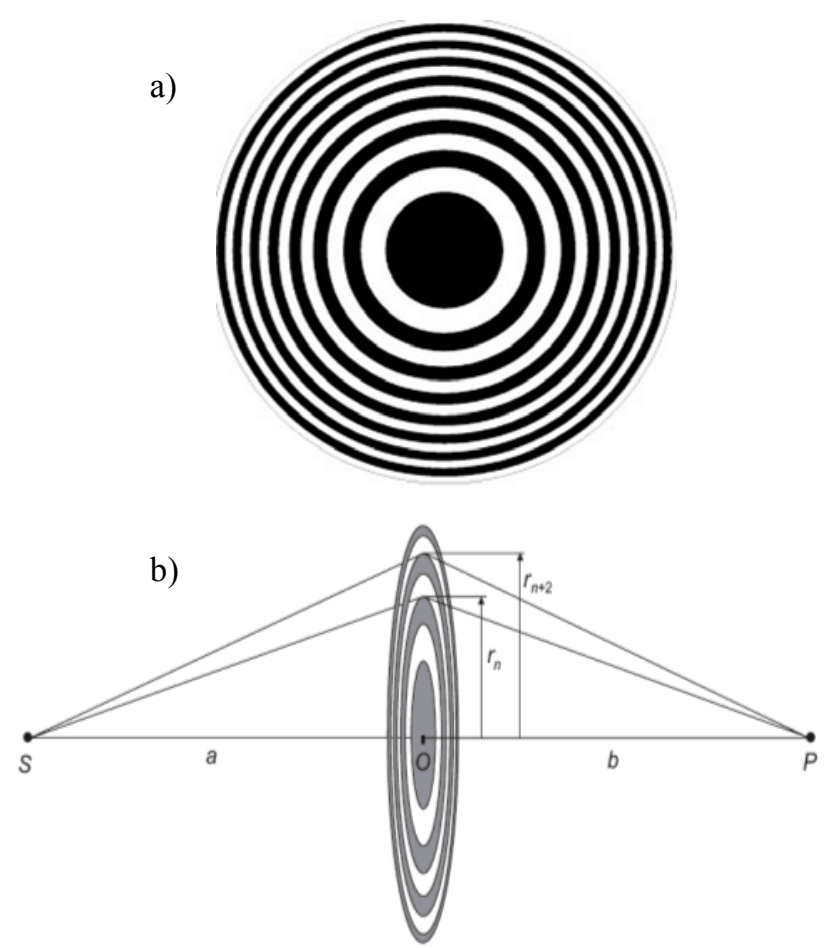

Fig. 1 a) Schematic diagram of a Fresnel zone plate and b) the geometry of a zone plate used for focusing.

\section{Experimental}

We first designed a "traditional", band-pass type FSS using a Jerusalem cross pattern etched into copper on polyimide. This structure was modeled using the Periodic Method of Moments, assuming a periodic boundary condition and infinite extent. Once the infinite Jerusalem cross pattern was optimized for the desired frequency $(40 \mathrm{GHz})$, this pattern was convolved with the pattern for a Fresnel zone plate, designed to focus at an appropriate distance (approximately 10 inches or $25 \mathrm{~cm}$ ). Examples of these patterns are shown schematically in figures $2 \mathrm{a}, 2 \mathrm{~b}$, and $2 \mathrm{c}$, respectively. 
a)

b)

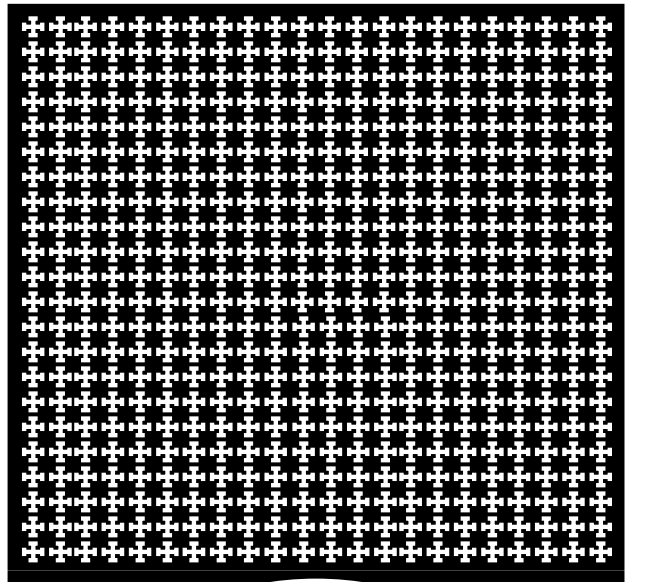

c)

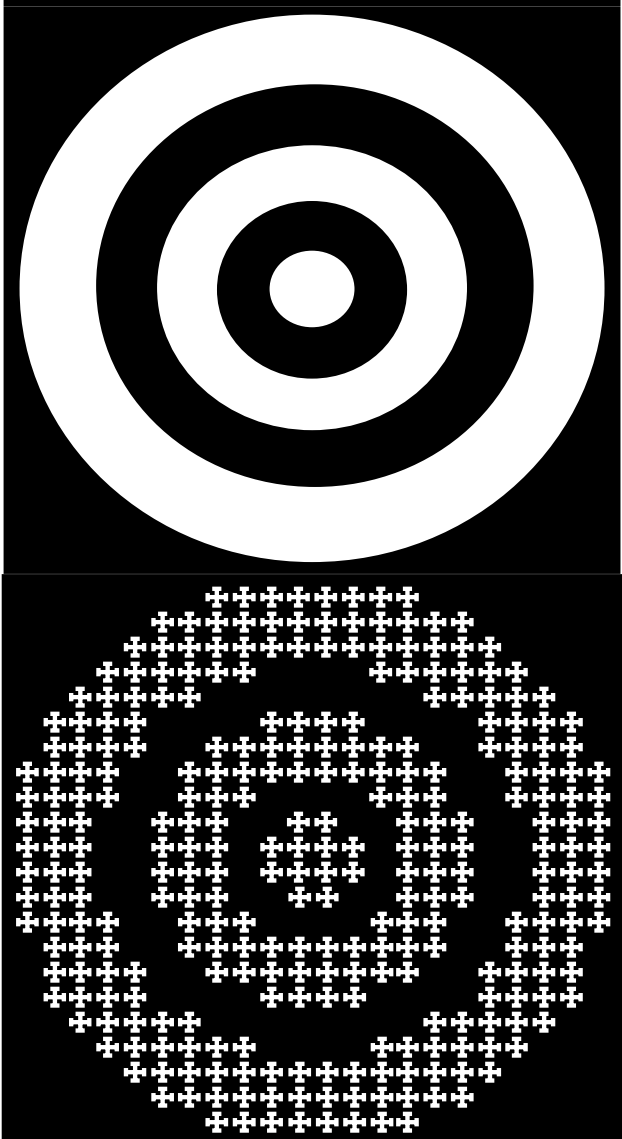

Fig. 2 Schematic representations of a) a "traditional" FSS, b) a Fresnel zone plate, and c) a convolution of the two patterns.

The prototype lens was fabricated using nonlithographic, laser patterning on a sheet of copper clad polyimide ( $11 \mu \mathrm{m}$ copper on $25 \mu \mathrm{m}$ polyimide). The copper side of the sheet was coated with S1818 photoresist (Shipley-Microposit, Marlborough, MA) using a \#4 wire wound applicator (Leneta Inc., Mahwah, NJ). The sample was baked at $120^{\circ} \mathrm{C}$ for 15 minutes to dry the S1818. A custom built laser micro-machining system was used to pattern the S1818 layer by laser ablation. Details of the laser ablation system are given elsewhere [12]. The system used a 266 $\mathrm{nm}$, solid state laser with $20 \mathrm{nsec}$ pulse length and $30 \mathrm{kHz}$ pulse repetition rate. The use of a galvanometric beam scanner, combined with XY translation stages allowed the full area of the part $(180 \mathrm{~mm}$ by $180 \mathrm{~mm})$ to be patterned in approximately 1 hour. The part was then submerged in a standard PCB etching solution (ferric chloride) and agitated for approximately 15 minutes to remove the copper from the exposed areas. The part was then rinsed with tap water and the S1818 layer was removed with acetone. A photograph of the completed prototype lens is shown in figure $3 \mathrm{a}$. The overall size of the substrate is $180 \mathrm{~mm}$ by $180 \mathrm{~mm}$, and the diameter of the outermost circular zone (which defines the diameter of the lens) is $176 \mathrm{~mm}$. A detailed view of the Jerusalem crosses is shown in figure $3 \mathrm{~b}$. Each cross is $2 \mathrm{~mm}$ by $2 \mathrm{~mm}$, with a $1 \mathrm{~mm}$ "tee" at the end of each arm. The line width varies from about 175 to 200 microns.

a)
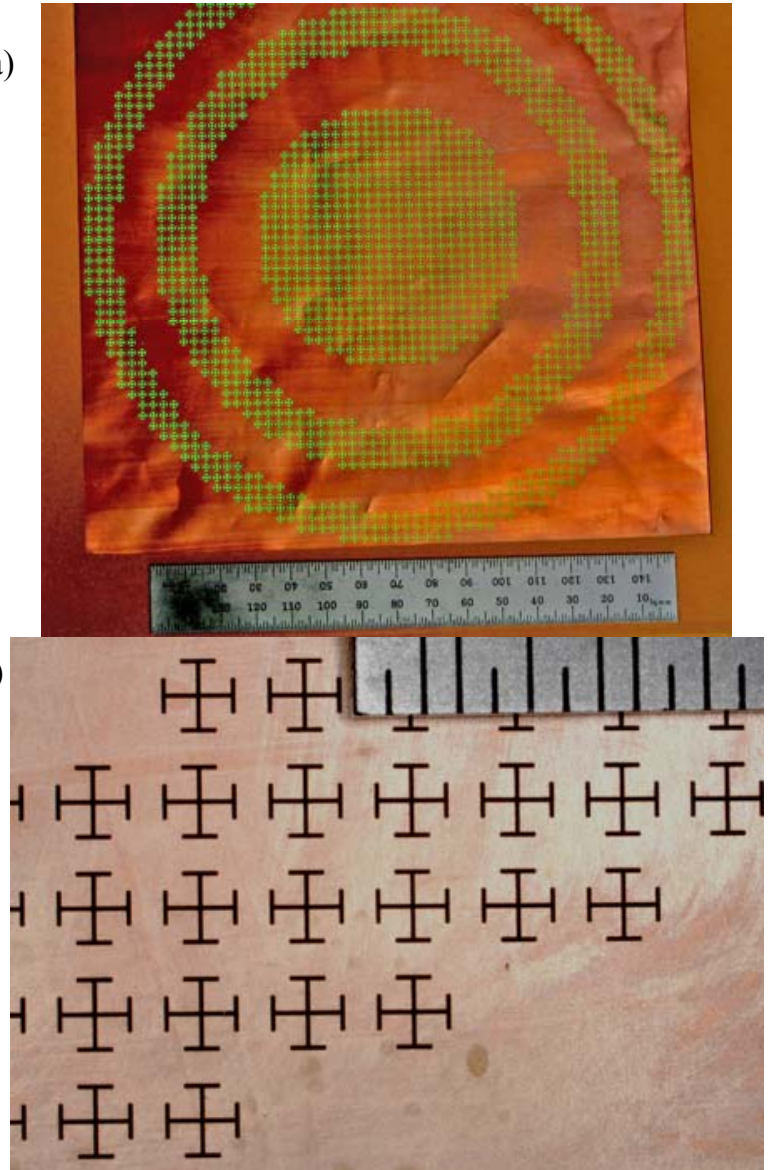

Fig. 3 Photograph of a Fresnel zone plate lens, showing a) the complete lens, backlit with green light and b) a closeup of the patterned elements. (Both scales in millimeters)

The electromagnetic properties of the lens were measured in an anechoic chamber using an Agilent E8363B PNA network analyzer and a motion control system with a LabView $^{\text {tm }}$ interface. The transmitter was a rectangular horn antenna producing nearly plane-wave illumination at the sample. The receiver was a rectangular waveguide antenna, with no horn attached. The sample was placed at a fixed distance from the transmit horn with center of the lens aligned with the center of the transmit horn (i.e. optical axis of the lens coincident with the direction of propagation of the plane wave). The receive antenna was placed at various distances from the part, also aligned with the center of the lens. At each lens/receiver distance, the lens was removed and the network analyzer was calibrated. The lens was then re-inserted in the same position, using fixed alignment pins. The transmittance was measured as a func- 
tion of frequency from 30 to $45 \mathrm{GHz}$, at each lens/receiver distance. A schematic diagram of the system is shown in figure 4.

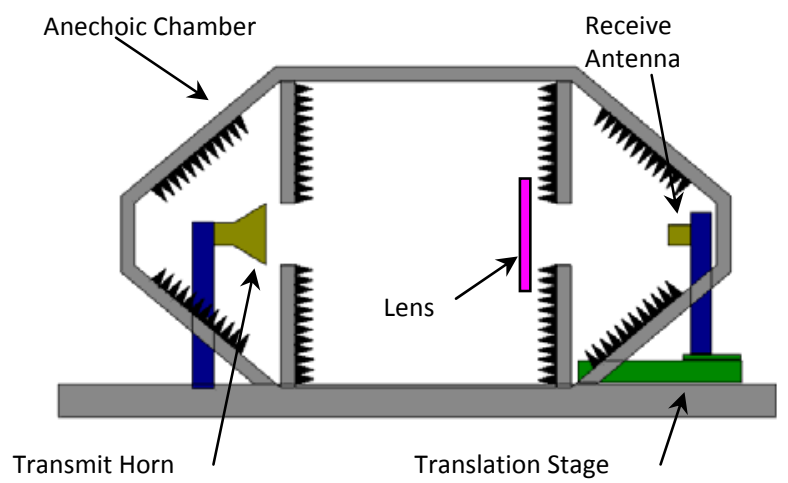

Fig. 4 Schematic diagram of the measurement system used in combination with an Agilent E8363B network analyzer.

\section{Results}

The measured transmittance of the lens is shown in figure 5a. This measured transmittance is compared with the predicted transmittance shown in figure $5 \mathrm{~b}$. Note that the predicted behavior is not determined by modeling the actual structure, but rather results from the convolution of a PMM simulation of an infinite FSS combined with the performance of a non-frequency selective Fresnel zone plate. The direct modeling of this structure would require a "mesh" fine enough to capture the behavior of the individual Jerusalem crosses (on the order of a few microns) and large enough to capture the behavior of the Fresnel zone plate (a $200 \mathrm{~mm}$ by $200 \mathrm{~mm}$ area). A "mesh" containing this many elements would be unwieldy for most computers. As a result, we resort to simulating the two structures independently, and then estimating the performance of the complete structure as the convolution of the two simplified models.

It is clear from figure 5 that reasonable agreement exists between the predicted and measured behavior of this lens. There are, however, noticeable differences. Although some of these differences may be due to variation in line width (a result of the copper etching process), we attribute most of the discrepancy to the "convolution of models" approximation. The predicted behavior, shown in figure $5 \mathrm{~b}$, results from the convolution of two separate modeling steps; modeling an infinite FSS and modeling a circular Fresnel zone plate. In reality, each zone of the Fresnel zone plate has a "jagged" edge, due to the discrete nature of the FSS pattern. As a result of these non-circular zones, we would expect the simulated data to underestimate the Raleigh range of the lens: i.e. the model should show a very sharp focus with respect to changes in distance, while the actual sample should show some "blurring". This behavior is consistent with the results shown in figure 5 .

The transmittance of the lens is measured to be as high as 35 , implying that the lens causes 35 times more intensity to fall onto the receive antenna, as compared to the intensity without the lens.
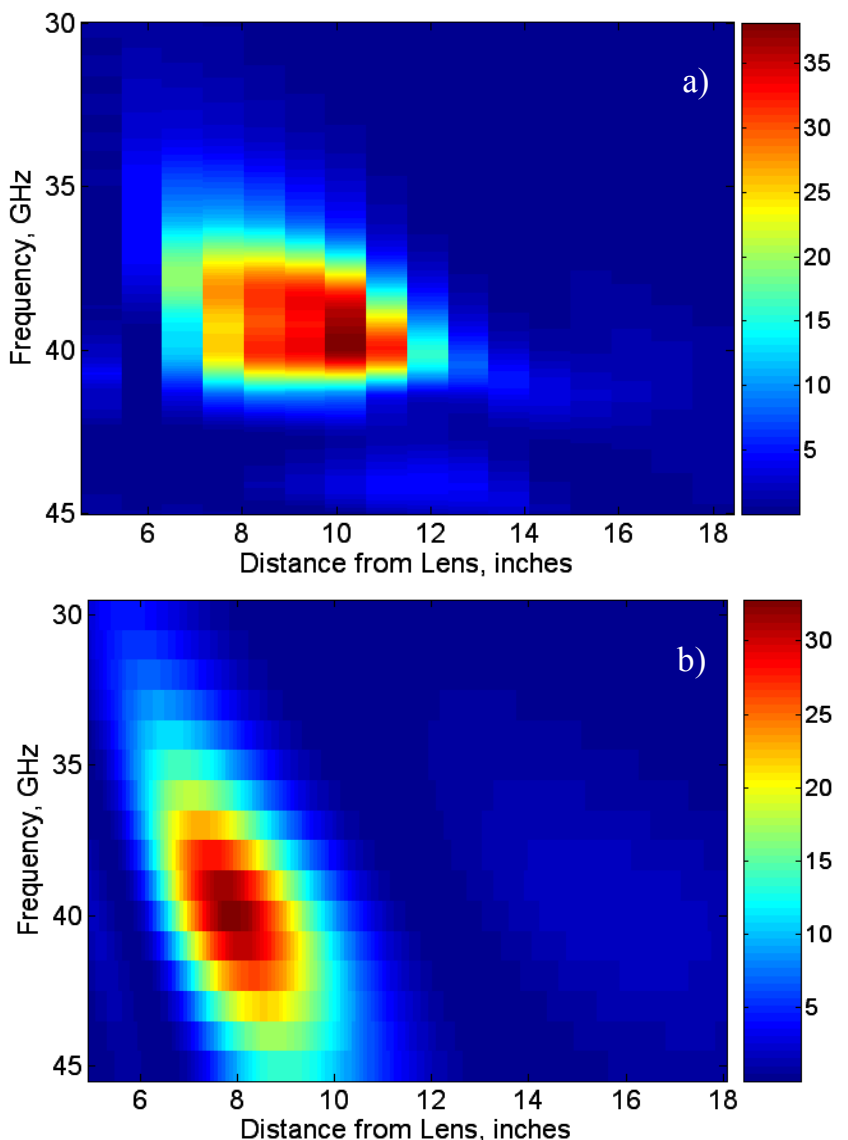

Fig. 5 a) Measured and b) calculated transmittance of the lens as a function of frequency and lens/receiver distance.

The data in figure 5a show that the prototype sample is acting as a lens. It is clearly focusing electromagnetic energy at a distance of approximately 10 inches $(25 \mathrm{~cm})$. Additionally, these data indicate that the prototype sample only focuses a narrow band of frequencies, centered between 37 and $41 \mathrm{GHz}$

Although we were able to predict the behavior of this structure a priori, it is worth noting that this frequency selective lens is a rather simple, aperiodic design. As a result, it is relatively easy to predict its behavior without the numeric modeling of the complete structure. The electromagnetic behavior of other, more complicate or less symmetric structures cannot be predicted so easily. Even in cases where a "convolution of models" allows one to predict the performance of a "non-traditional" FSS, it is clear that this approximation becomes less exact and less reliable when applied to highly aperiodic structures. Rapid prototyping techniques, such as laser direct-write, offer a clear advantage over conventional lithographic fabrication for the design of aperiodic or spatially varying electromagnetic structures.

\section{Conclusions}

We present the design and fabrication of a frequency selective Fresnel zone plate lens using a laser-based, nonlithographic, rapid prototyping process. We demonstrate that the prototype device focuses electromagnetic energy and exhibits band-pass behavior, as predicted. We conclude that laser based rapid prototyping plays a crucial role 
in the development of novel RF and millimeter wave devices that rely on aperiodicity and spatially varying patterns to achieve new and useful electromagnetic properties.

\section{References}

[1] B.A. Munk, "Frequency Selective Surfaces; Theory and Design", (John Wiley and sons, New York, 2000)

[2] R. Remski et al., "Frequency Selective Surfaces", http://www.ansoft.com

[3] J. Romeu and Y. Rahmat-Samii: IEEE Trans. On Antennas and Prop. 48, (2000) 1097

[4] E.A. Parker, A.D. Chuprin, R.J. Langley: Proc. IEE, Microw. Antennas and Propag. 146, (1999) 319

[5] C.B. Arnold, P. Sera, and A. Piqué: MRS Bulletin, 32, (2007) 23

[6] S.A. Mathews, M.Mirotznik, B.L. Good, and A. Piqué: Proc. of SPIE, 6458, (2007) 64580R-1

[7] A.J. Mackay, "The mathematical formulation of QDAS, Q-par Dichroic Array Software", http://www.q-par.com/capabilities/research/fsssoftware-formulation

[8] Eugene Hecht, "Optics, $4^{\text {th }}$ Edition" (Addison Wesley, Reading MA, 2002) 485-496

[9] S. M. Stout-Grandy, J.S. Wight, A. Petosa, I.V. Minin and O.V. Minin: Microwave Journal, 51 (2008) "web exclusive"

[10]R. Ludewig, A. Garcia Ortiz, T. Murgan, J. Jesus, O. Hidalgo, M. Glesner: 14th IEEE International Workshop on Rapid System Prototyping, (2003) 172

[11] S.A. Mathews, M.Mirotznik, B.L. Good, and A. Piqué: Proc. of SPIE, 6458, (2007) 64580R-1

[12] A. Piqué, R.C.Y. Auyeung, H. Kim, K.M. Metkus, S.A. Mathews; Jour. of Laser Micro/Nanoeng. 3 (2008) 163

(Received: July 11, 2009, Accepted: October 26, 2009) 